\title{
FUZZY DECISION SUPPORT IN DISTRIBUTED V2V SYSTEMS
}

\author{
Aleksandr Fedorov, Sergey Zobnin, \\ Viacheslav Shkodyrev, Egor Goloschchapov,Vyacheslav Potekhin
}

Peter the Great St.Petersburg Polytechnic University, 29, Polytechnicheskaya st., St.Petersburg, 195251, Russia

\begin{abstract}
This paper presents a new approach of automated decision making in vehicle-to-vehicle (V2V) environments. Approach is based on extension of fuzzy knowledge-based decision making with automatic V2V communication that provides an ability for systems of Intelligent Transportation Agents (ITAs) to create a distributed network, where each agent is a part of the coalition that reacts to the environment changes (such as traffic jams, car accidents etc.). This paper considers a particular task of finding the shortest path in dynamical environments. An architecture of the system as well as mathematical model and simulation results is presented in the paper.
\end{abstract}

Keywords: distributed system; knowledge base; decision support; uncertancy; fuzzy
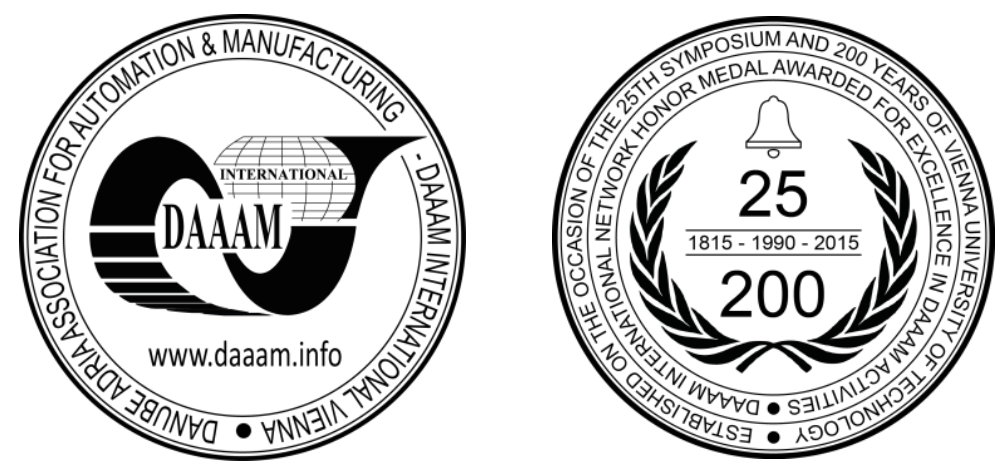

This Publication has to be referred as: Fedorov, A[leksandr]; Zobnin, S[ergey]; Shkodyrev, V[iacheslav]; Goloschchapov, E[gor] \& Potekhin, V[yacheslav] (2016). Fuzzy Decision Support in Distributed V2V Systems, Proceedings of the 26th DAAAM International Symposium, pp.0097-0103, B. Katalinic (Ed.), Published by DAAAM International, ISBN 978-3-902734-07-5, ISSN 1726-9679, Vienna, Austria DOI:10.2507/26th.daaam.proceedings.014 


\section{Introduction}

For a last decade in researchers and engineers areas the words "vehicle-to-vehicle network" becomes more and more popular. Moreover, with popularity of this idea the number of papers and researches grows every year. The main goal of this idea is to increase the safety on the roads and in ideal minimize it to zero [1]. Through the exchange of messages from vehicle to vehicle in some area can be established a mutual awareness, so any vehicle can change their route to a better road or avoid jam. Recently a small research group of inter-vehicle communication becomes a large consortium of researchers from the all over the world with support of biggest companies in machine industry. The area of research becomes start to cover many directions from a machine route optimization to awareness of cars with dangerous cargo.

In this paper represented use cases, the architecture and mathematical model of the system under development that combines two areas - fuzzy knowledge-based decision making and v2v communication. The both areas are distributed in terms that they not using centralized server and each vehicle does not know about every vehicle. Each ITA(Intelligent Transportation Agent) interacts with other ITAs in some area and transmits a knowledge and decisions given the data about situation on the roads.

\subsection{Applications}

The application of $\mathrm{v} 2 \mathrm{v}$ communication considered in this paper are distributed optimization of routes - finding the shortest path in dynamic environment and situation awareness based on distributed interactions between ITAs.

Distributed optimization of routes viewed in terms of finding the path between two points in an undirected weighed graph. In paper [2] introduced a comparison of variety of algorithms and theirs advantages and disadvantages that can be applicable for the system described in this paper. The system should react in real-time or near real-time to the changes in environment, because of this restriction, not all algorithms are applicable for the future system.

The second application - situation awareness viewed as a knowledge base with fuzzy rules that can produce the inference of rules and the environment state as described in paper [3]. All ITAs can produce a new knowledge - rules and if the same rule produced more frequently this rule will be more valuable for all ITAs.

Therefore, the system can combine the power of algorithms in area of more classical problem of finding the shortest path in a graph with extension to the dynamic weights and evaluation of rules that can be proceeded from environment.

\section{System description}

\subsection{Intelligent transportation agent}

In general, each agent divided into two parts - the decision making and knowledge base modules. Knowledge base is responsible for storing the rules and evolving based on new data perceived not only from the ITA(local knowledge base) but also from other ITAs (distributed knowledge), the same is true for a decision making module which is responsible for a situation assessment and path optimization, see Fig. 1.

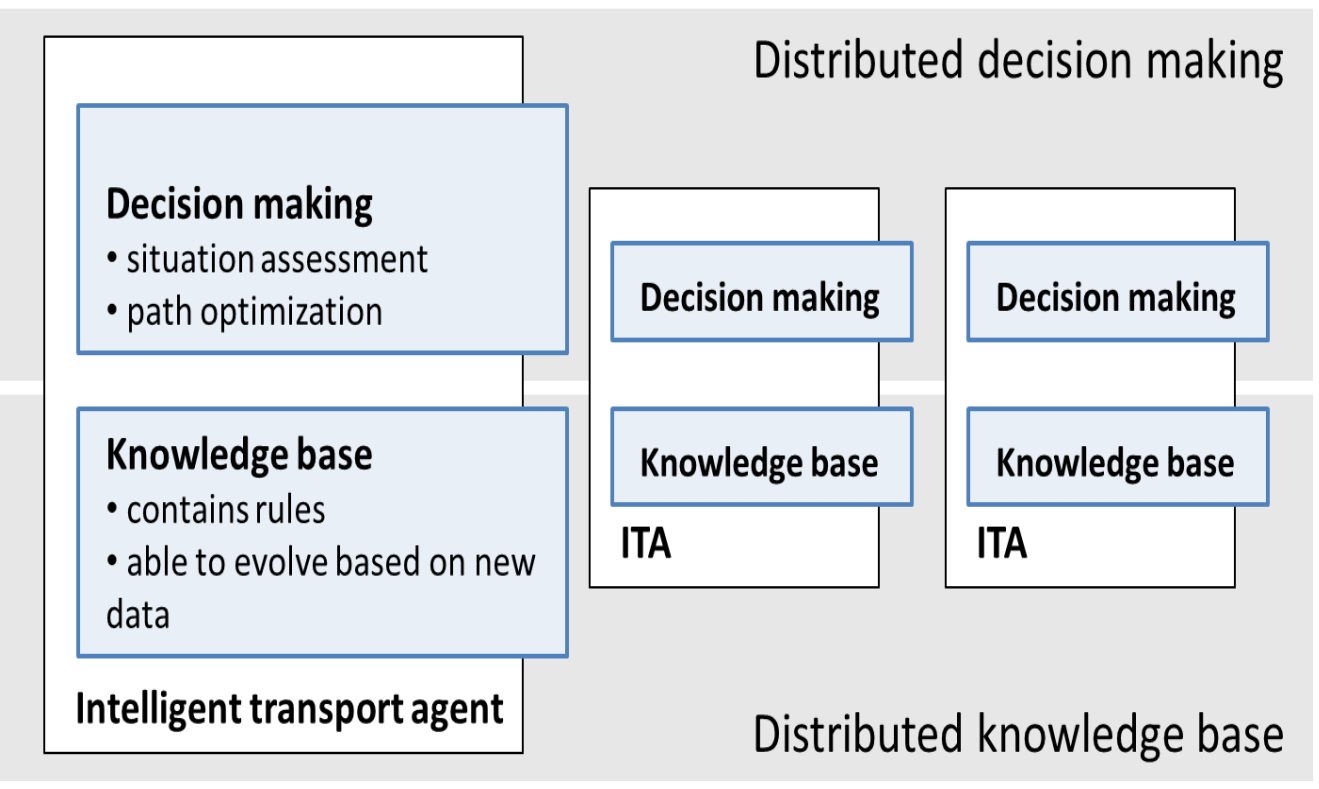

Fig. 1. Intelligent Transportation Agent architecture 
The main features of the v2v system under development are:

- Agents exchange knowledge rather than raw data. Each agent has an evolving knowledge base, common space of concepts.

- Distributed output of fuzzy rules from the statistics - agents transmits new rules, calculating the core of "the most truthful and popular rule"

- $\quad$ New rules get even more weight in decision-making

- Predetermined rules may be removed from the decision circuit according to the new information from agents

\subsection{Knowledge base}

The knowledge base that we are developing has some classical properties and some of the features that allow distinguishing our approach from other developments in this field:

1. Real-time knowledge transfer as shown on Fig. 2. All ITAs can generate a new rule and transfer this rule to the other ITAs, allowing them to consider this rule when make a decision in current environment state.

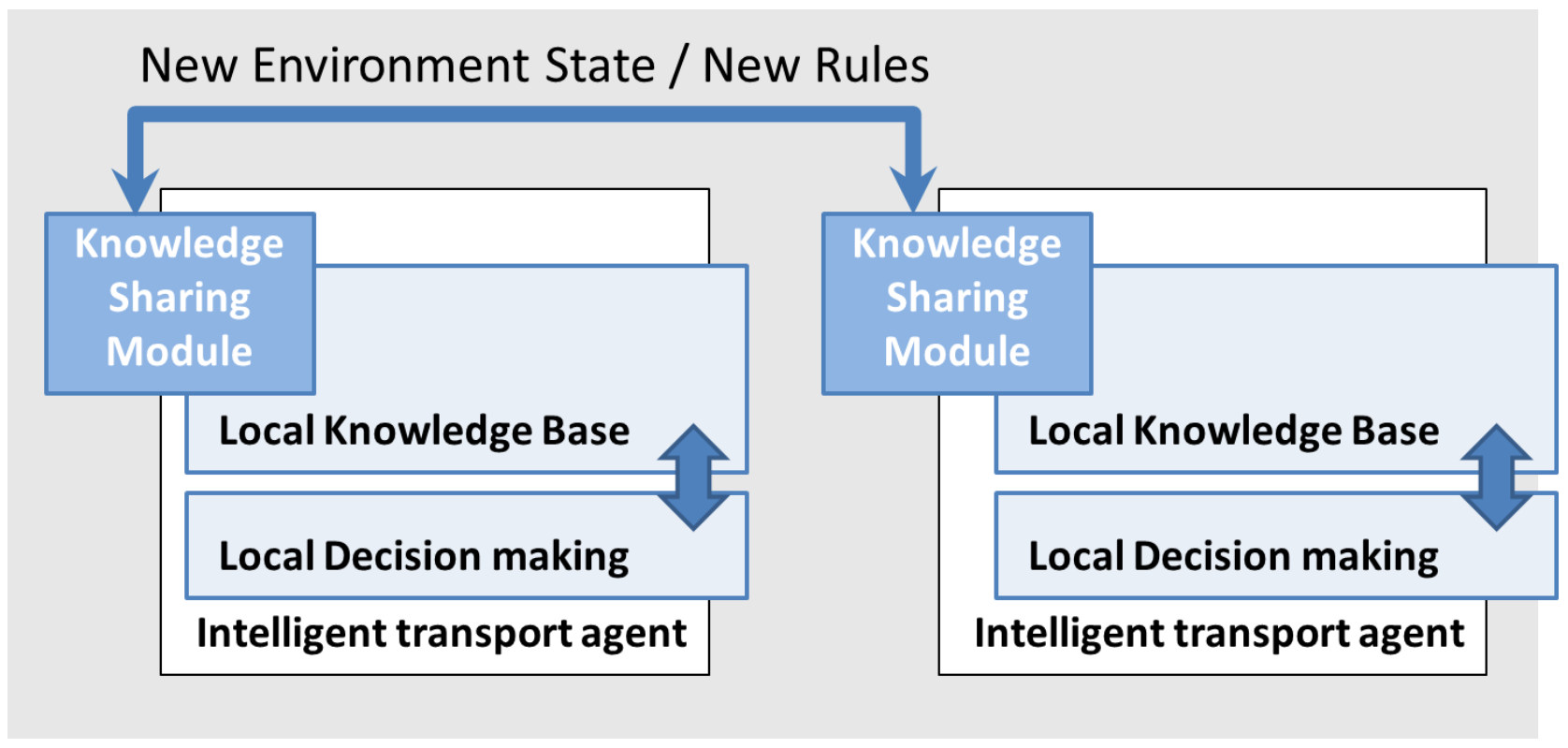

Fig. 2. Distributed Knowledge Based Decision Making

2. Generation of new rules. For example, if there are 10 cars near semaphore, the traffic jam appeared in 60 cases out of 100 , so can be generated a new rule.

3. Evolution of old rules. The rule can be constantly updated, let us say in 1,000 cases the traffic jams will appear 650 times and that will change the weight of the rule. In rule can also vary the number of cars. Example of evolution of rules in multi agent system proposed by authors in [4].

4. Inference is performed based on distributed and local knowledge. The local knowledge and distributed knowledge, which is transferred from other ITAs, will be merged if the rules has equal terms and variables. For example rule "If car is broken the traffic jam probability will be High" and rule "If number of cars near semaphore a 10 then traffic jam probability will be Medium" will be merged to rule "If number of cars is 10 and car ahead is broken then the probability of traffic jam will be Very High".

The basic approach of distributed knowledge base is described in paper [5] and [6].

The example of work of two ITAs with deferent layers of knowledge base can be viewed on Fig. 3 - agent that close to the traffic jam can recognize the number of cars below the semaphore and given the rule "If the number of cars near the semaphore more than $\mathrm{N}$ then the probability of traffic jam is High". The $\mathrm{N}-$ the number of cars can be transmitted to the nearest agent and given this value, ITA can decide to turn in other way to find a better path. 


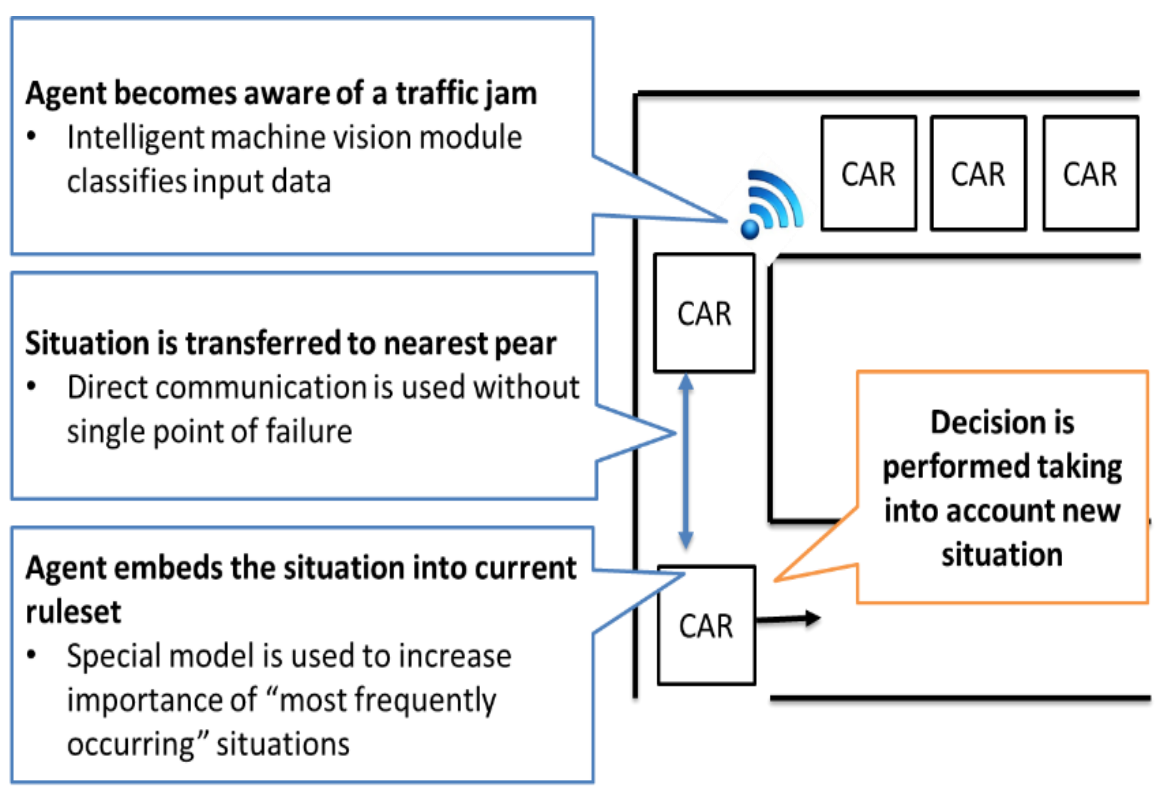

Fig. 3. Distributed Knowledge Based Decision Making

\section{Mathematical model}

The mathematical model consist also from two parts - knowledge base part with inference of logical rules and shortest path solver for graph with dynamic weights.

Let us denote for finding the shortest path the $G=(V, E)$ - city graph, where vertices represent street crossings or other special objects, edges are roads between street crossings.

Let $w_{i, j}$ be a weight of edge $e_{i, j}$ which in our case consists of a static weight $s w_{i, j}$ and a dynamic weight multiplier $d w m_{i, j}$.

$w_{i, j}=s w_{i, j} * d w m_{i, j}$

Static weight $s w_{i, j}$ is a weight determined from graph structure (lengths of roads). Dynamic weight multiplier $d w m_{i, j}$ is inferred in runtime from knowledge base. Let $\boldsymbol{S}$ be a set of possible situations that ITA can be aware of $|\boldsymbol{S}|=$ $k$. For each possible situation, there is a coefficient $\alpha_{k}$ which represents how much the situation $s_{k}$ affects the weight $w_{i, j}$. Situation itself is an output variable of fuzzy set. Each situation can be in three fuzzy states: LOW, MEDIUM and HIGH (there can be more states but for simplicity were chosen three states). Membership function $\mu\left(s_{k}\right)$ shows if ITA is in particular situation.

$d w m_{i, j}=\sum_{k} \mu\left(s_{k}\right) * \alpha_{k}$

The logical structure of the mathematical model shown on Fig. 4:

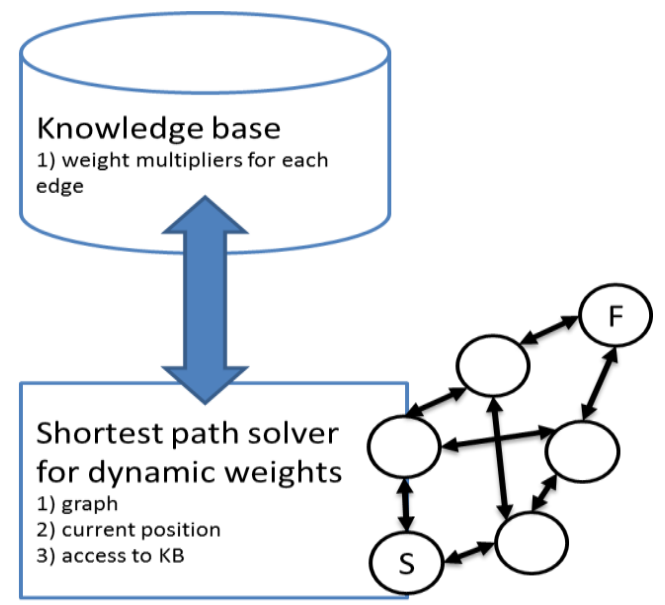

Fig. 4. Distributed Route Optimization of ITA 
For finding the shortest path from point $S$ to point $F$ each ITA uses Dijkstra's algorithm and the knowledge base. With the transition to the next point of graph, ITA recalculates weight of edges that allows dynamically avoiding the problem areas and moving faster as a result. The system can use not only Dijkstra's algorithm but also the $\mathrm{A}^{*}$ or another algorithms for finding the shortest path in graph.

\section{Prototype implementation}

Following general concepts presented in previous chapters, we developed an architecture of Intelligent Transportation Agent, which is presented in Fig. 5.

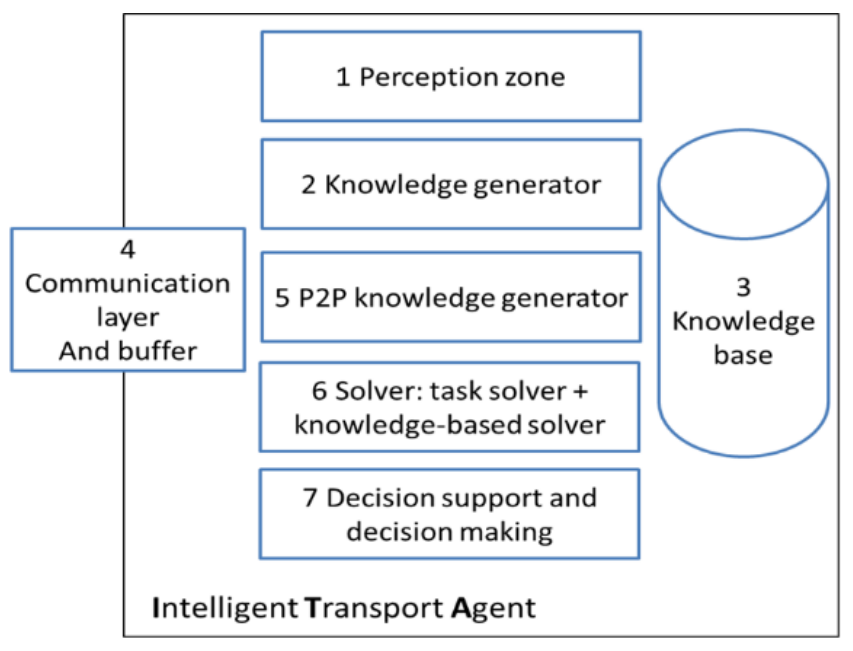

Fig. 5. Architecture of Intelligent Transportation Agent

According to this architecture, every implementation of ITA concept should contain the following components/functionalities:

1. Perception zone

2. Knowledge generator

3. Knowledge base

4. Communication layer/component

5. $\mathrm{P} 2 \mathrm{P}$ (peer-to-peer) knowledge generator

6. Solver

7. Decision support and decision making component

In order to evaluate maintainability and portability of the resulting architecture right during the development phase, we have implemented ITA concept in a simulated environment as a part of architecture development. Practically both ITA implementation and environment are written in Java and are executed in same process on a Windows/Linux machine. Development approach and techniques that we are using allow changing implementation of perception zone and communication component (\#1 and \#4 respectively in Fig. 5) in order to achieve physical distribution and deploy ITA implementation in a physically distributed computational environment (i.e., VANET [7]). Resulting environment modeling algorithm and ITA lifecycle are presented in Fig. 6.

ITA lifecycle generally bounds all components presented in Fig.5 into single process of transforming incoming two incoming data flows (sensor data from environment on line 2 and messages from other ITAs on line 4) into a decision. In our particular implementation decision, generation and implementation are separate processes; such separation allows increasing maintainability and flexibility of the simulation. Implementation of decision that causes a possible change of ITA state (an explicit data structure designed in object-oriented form, ITA state is a reference to a particular coordinate in global road network with additional parameters like speed, position, direction, etc.) is initiated by environment lifecycle. Local knowledge base is updated on lines 3 (where KB update is caused by new local observations) and 4 (where $\mathrm{KB}$ update is caused by messages from other ITAs via mechanism of distributed awareness).

The knowledge base transforms incoming data into inference engine input variables and binds those variables with fuzzy logic rules. Then KB infers rules outputs and calculates the multiplier coefficients for a decision maker as presented in previous chapter. Takagi-Sugeno method is used to perform inference, because it suits the use case of solving problems with multiple inputs and outputs (MISO, MIMO), and usually provides better results than other methods. One of alternative methods is Mamdani, which is commonly is used in simple problems with two inputs and one output [8]. 

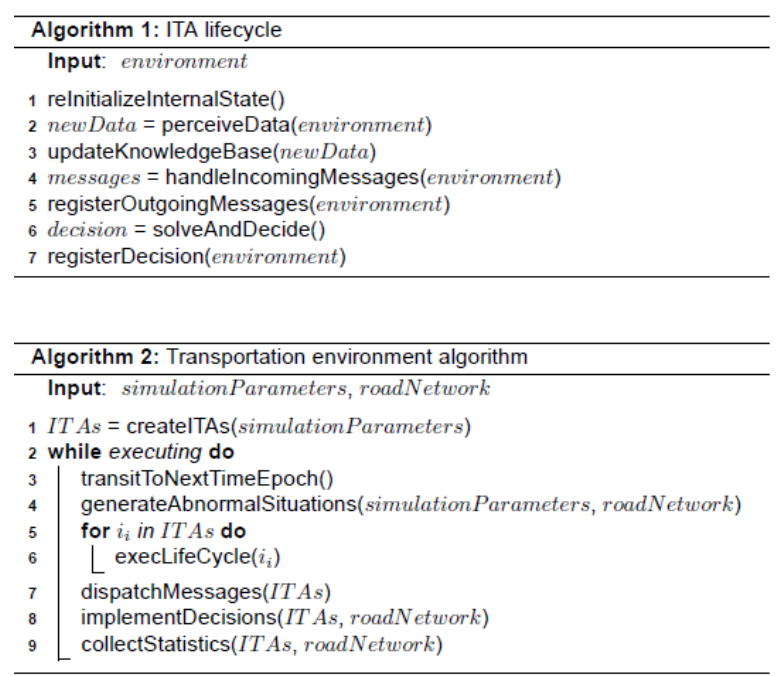

Fig. 6. Algorithms of ITA and environment

In our simulation ITA solve tasks of achieving their desired ending states under pressure of randomly occurring traffic jams. Ending state is a reference to a position on a global road network and ITA moves through a set of roads to achieve the ending state. On line 6 of ITA lifecycle an actual call to a problem solver is performed. In our simulation, we consider different options for solving the weighted shorted path problem. First, a standard Dijkstra's algorithm is used to compute the shortest path according to static information about road lengths. Second, a dynamic algorithm that recomputes optimal path in each ITA lifecycle call based on internal knowledge base via mechanism presented in the previous chapter.

Environment lifecycle is the main event loop of simulation, which bounds multiple ITAs into single logical space where agents are able to communicate and perceive local data, as well as change their state according to generated decisions, which in turn are generated based on local and distributed knowledge. During the simulations abnormal situations are generated with some degree of randomness (line 4 in Transportation environment algorithm), in our case abnormal situations are traffic jams. Environment is also responsible for message dispatching and decision implementation initiation (lines 7-8). Last, but not the least, environment collects performance statistics on line 9.

Approaches and technology described in article [9] will be applied to the study of this article, so that the system can take advantage of a distributed system - scalability, fault tolerance and load balancing.

\section{Results and further work}

In this chapter, we briefly summarize results and findings that were acquired during our research. We also draw reader's attention on some implications of our particular design and our experiments.

First, we have developed a concept of application of distributed knowledge base technology in the domain of logistics (vehicle-to-vehicle environments). The concept converged to ITA (Intelligent Transportation Agent) with particular contents, architecture and functionalities. We conclude that the developed approach exceeds our initial expectations because it allows optimizing criteria and tasks that we were not expecting to consider.

Second, according to a concept we have developed a mathematical model that integrates knowledge-based decision making with concrete problem solving technique applied to a problem of optimal route finding in traffic jam environments. Mathematical model with a detailed architecture of ITA yields a set of developed algorithms for ITA that are also presented in this paper.

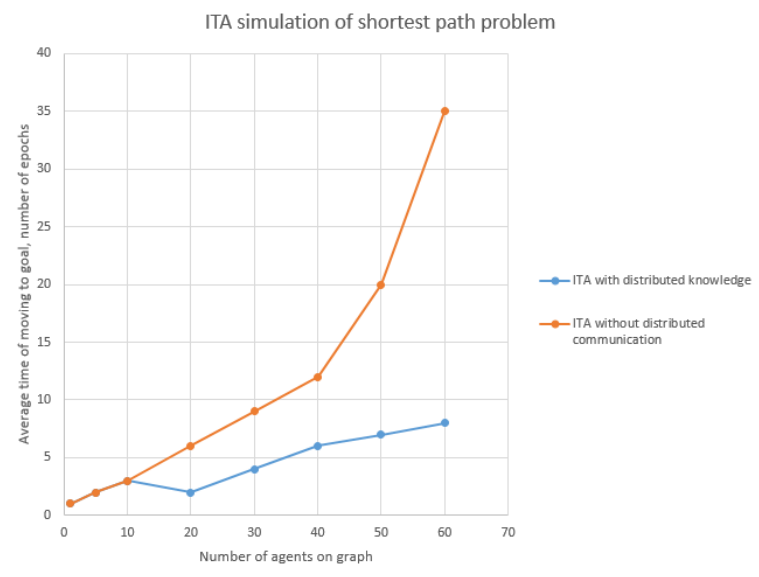

Fig. 7. Simulation results of finding shortest path by ITA in dynamic environment 
Third, we have developed a software simulation to prove a concept. The simulation results of solving the problem of the shortest path by ITA shown on Fig. 7. In the simulation, we compared our approach with standard optimal route finding via Dijkstra algorithm. For a given road network we executed both approaches and averaged results. Y-axis of the chart is a number of "epochs" - virtual time steps that are used to track time inside the simulation. We conclude that our results corresponds to expectations in this particular problem and explain the chart's data in the following way. While standard shortest path solutions can not handle traffic jams efficiently, ITA-based approach with communication and knowledge-based decision making yields shorter times of reaching destinations without any centralized infrastructure (a server that will collect the data from all automobiles and dispatch the decisions) which means that optimal route finding via our approach may be performed in highly dynamic environments or road networks that do not contain all required centralized infrastructure.

We consider the following problems for ITA's to consider on the next stages of our research:

- Support of special transport and transport with dangerous cargo

- Organization of corridors of free movement for special transport

- Distributed optimization search of parking spaces in a distributed system

- Optimization of supply chain management

- Increasing of the total capacity of the transport network / decrease in average travel time

\section{Conclusion}

The safety on the road based on vehicle-to-vehicle communication has motivated this paper research to study new approaches to solve the problem of continuous changes on the road. The developed approach can work with all types of sensors even if the universal communication protocol of IEEE family still not finished. Vehicles in this paper supposed to operate in intelligent and self-organizing manner. Moreover, to perform this scenario discussed the approach of distributed knowledge and decision-making. Considered the new algorithm that consist of some classical approaches with new features such as dynamical evolving rules and decision making based on these rules and solving the shortest path problem. Described approaches can be applied in city transportation system to increase safety and decrease load on roads. The simulation results shows the advantage of distributed model when each ITA communicate with each other. Should be considered that in research were looked specific cases, when all cars have the same map (in our case the graph), as well as the types of contingencies, and the road itself consisted only of intersections. In further work planned to expand the set of rules and increase complexity of the road taking into account the cars not connected to the distributed ITAs network. Also in described example not taken into account the velocity of the cars and their types.

\section{References}

[1] J. Whitelegg, G. Haq, Vision Zero: Adopting a target of zero for road traffic fatalities and serious injuries. Stockholm, Sweden: Stockholm Environment Institute, 2006.

[2] H. Bast, et al., Route Planning in Transportation Networks,Technical report, MSR-TR-2014-4, Microsoft Research, 2014.

[3] D. Hládek, Learning System Based on Generalization of Fuzzy Rules, PhD thesis, Technical University of Kosice, 2009.

[4] H. Wang, S. Kwong, Y. Jin, W. Wei, K. F. Man, Agent-based evolutionary approach for interpretable rule-based knowledge extraction. Systems, Man, and Cybernetics, Part C: Applications and Reviews, IEEE Transactions on, 35(2), 2005, pp.143-155.

[5] D. A. Carlson, S. Ram, An architecture for distributed knowledge based-systems, ACM SIGMIS Database, №. 12., 1991, pp. 11-21.

[6] A. Fedorov, S. Zobnin, V. Shkodyrev, Knowledge Based Planning Framework for Intelligent Distributed Manufacturing Systems, Advances in Swarm and Computational Intelligence. - Springer International Publishing, 2015, pp. 300-307.

[7] H. R. Arkian, R. E. Atani, A. Pourkhalili, S. Kamali, A stable clustering scheme based on adaptive multiple metric in vehicular ad-hoc networks. Journal of Information Science and Engineering, 31(2), 2015, pp. 361-386.

[8] K. Guney, N. Sarikaya, Comparison of Mamdani and Sugeno fuzzy inference system models for resonant frequency calculation of rectangular microstrip antennas, Progress In Electromagnetics Research B, 2009, pp. 81104.

[9] S. Zobnin, A. Fedorov, E. Goloschchapov, O. Ipatov, V. Potekhin, V. Shkodyrev, Aspects of Smart Manufacturing Via Agent-based Approach. Procedia Engineering, 100, 2015, pp.1572-1581. 